\title{
Evaluating respiratory musculature, quality of life, anxiety, and depression among patients with indeterminate chronic Chagas disease and symptoms of pulmonary hypertension
}

\author{
Alícia Cristina Suman ${ }^{[1]}$, Érika Alessandra Pellison Nunes da Costa ${ }^{[1],[2],}$ \\ Silméia Garcia Zanati Bazan ${ }^{[3]}$, João Carlos Hueb ${ }^{[3]}$, Fabio Cardoso de Carvalho ${ }^{[3]}$, \\ Luis Cuadrado Martin ${ }^{[3]}$ and Hugo Hyung Bok Yoo ${ }^{[3]}$
}

[1]. Programa de Pós Graduação em Doenças Tropicais, Faculdade de Medicina de Botucatu, Universidade Estadual Paulista, Botucatu, SP, Brasil. [2]. Departamento de Doenças Tropicais e Diagnóstico por Imagem, Faculdade de Medicina de Botucatu, Universidade Estadual Paulista, Botucatu, SP, Brasil. [3]. Departamento de Clínica Médica, Faculdade de Medicina de Botucatu, Universidade Estadual Paulista, Botucatu, SP, Brasil.

\begin{abstract}
Introduction: Chagas disease (CD) is progressive and incapacitating, especially when cardiopulmonary function is affected. For example, respiratory muscle weakness can cause dyspnea upon exertion and fatigue, which may be exacerbated when it is associated with pulmonary hypertension $(\mathrm{PH})$. The present study aimed to evaluate respiratory musculature, quality of life, anxiety, and depression among patients with indeterminate chronic CD and symptoms of PH. Methods: All individuals completed a clinical evaluation, spirometry, a 6-min walking test, respiratory musculature testing using maximum inspiratory pressure (PImax) and maximum expiratory pressure (PEmax), the Hospital Anxiety and Depression Scale, and the SF-36 questionnaire. Results: We evaluated 107 patients who were assigned to a control group with only CD (G1, 8 patients), a group with CD and possible $\mathrm{PH}$ (G2, 93 patients), and a group with $\mathrm{CD}$ and echocardiography evidence of $\mathrm{PH}$ (G3, 6 patients). The three groups had similar values for PImax and PEmax. Compared to the G1 and G2 groups, the G3 group covered significantly less distance during the 6-min walking test and had a significantly shorter predicted distance ( $<<0.05$ vs. the G1 group). All three groups had similar values for their spirometry results, Hospital Anxiety and Depression Scale scores, and SF-36 questionnaire results. Conclusions: Patients with indeterminate chronic $\mathrm{CD}$ and symptoms of $\mathrm{PH}$ did not experience significant impairment in the studied variables, with the exception of the 6-min walking test, which suggests a low exercise tolerance.
\end{abstract}

Keywords: Chagas disease. Pulmonary hypertension. Quality of life. Respiratory musculature.

\section{INTRODUCTION}

Pulmonary arterial hypertension $(\mathrm{PAH})$ is a serious clinical condition characterized by vasoconstriction, thrombosis in situ, and vascular remodeling, which leads to progressive right ventricular insufficiency and eventually to death. The diagnosis is based on right cardiac catheterization with a resting mean arterial pressure of $>25 \mathrm{mmHg}$ and a pulmonary artery occlusion pressure of $<15 \mathrm{mmHg}^{1}$. The main symptoms of PAH are early fatigue and dyspnea, which can limit the individual's ability to perform their activities of daily living. These symptoms are associated with inefficient gas exchange and an inability of the right ventricle to augment the pulmonary blood flow and oxygen supply.

\footnotetext{
Corresponding author: Dra. Alicia Cristina Suman.

e-mail: aliciasuman@hotmail.com

Received 26 October 2016

Accepted 24 February 2017
}

The physical limitations of pulmonary hypertension $(\mathrm{PH})$ have attracted significant clinical interest, as they highlight the probable evolution of the disease. Muscle strength testing has revealed decreased levels of respiratory musculature, especially in the diaphragm, which is the primary muscle that drives respiration. Thus, decreased respiratory musculature may account for the dyspnea upon exertion and fatigue in patients with $\mathrm{PH}^{2}$ Disease severity and therapeutic response in $\mathrm{PH}$ cases is generally evaluated using quality of life questionnaires, such as the 36-item Short Form (SF-36) questionnaire ${ }^{3}$ Individuals with cardiocirculatory disorders can be evaluated using the 6-min walking test (6MWT), which is used as both a diagnostic tool and a tool to clinically evaluate the individual. Furthermore, the magnitude of the individual's limitations can be evaluated in terms of functional class, which is determined based on an examiner's assessment of the individual's ability to perform various physical exercises.

Chagas disease is considered a neglected disease by both governmental agencies and the pharmaceutical industry. This infection is caused by a flagellar protozoan (Trypanosoma cruzi) 
and is considered an important public health problem ${ }^{4}$, as the World Health Organization estimates that approximately 6-7 million people are currently infected ${ }^{5}$ Chagas disease typically progresses through three clinical stages: acute, chronic indeterminate, and chronic determinate. Approximately $95 \%$ of cases involve the acute phase, which is frequently asymptomatic but can provoke myocarditis and encephalomyelitis in $5-10 \%$ of cases; if untreated, the acute phase progresses to the indeterminate chronic phase ${ }^{6}$. Patients with indeterminate Chagas disease do not have a decreased cellular immune response and have normal findings during echocardiography and radiography of the heart, esophagus, and colon. Thus, the disease is only usually detected using positive serology results or detection of the parasite through xenodiagnosis, blood culturing, or polymerase chain reaction (PCR). Patients with indeterminate Chagas disease have low morbidity, are capable of performing any type of activity, and have an excellent prognosis for at least 5-10 years ${ }^{7}$.

Most patients with indeterminate chronic Chagas disease report difficulty in performing normal physical activities. Similar to patients with $\mathrm{PH}$, these patients' limitations are strongly associated with their quality of life. Chagas disease is prevalent in Brazil but remains neglected, and there is a scarcity of studies that have emphasized the possible relationship between physical functioning and quality of life. Therefore, the present study evaluated respiratory musculature among patients with Chagas disease, and the possible associations of indeterminate chronic Chagas disease with $\mathrm{PH}$ symptoms, quality of life, anxiety, and depression.

\section{METHODS}

This prospective study was conducted between March 2013 and February 2016. The sampled group included 300 patients who had been monitored in the Infectious Diseases Ambulatory Unit (Botucatu School of Medicine, State University of São Paulo). All subjects gave their written informed consent for the participation to the study.

Among these individuals, 187 had indeterminate chronic Chagas disease and were divided into three groups (Figure 1). The control group (G1 group) included asymptomatic patients. The G2 group included patients with clinical symptoms of $\mathrm{PH}$ but echocardiography findings that did not support the suspicion of PH. The G3 group included patients with clinical symptoms of $\mathrm{PH}$ and echocardiography findings that supported the suspicion of $\mathrm{PH}$. The presence or absence of $\mathrm{PH}$ in this group was confirmed using right cardiac catheterization. The exclusion criterion was patients who had cardiac, digestive, mixed, and other pathologies that could generate clinical symptoms that were suggestive of $\mathrm{PH}$.

The echocardiography findings were obtained and analyzed according to the recommendations of the American Society of Echocardiography. A systolic arteriopulmonary pressure of $\geq 40 \mathrm{mmHg}$ was considered suggestive of $\mathrm{PH}$.

All patients underwent a complete clinical examination, which included their medical history, a questionnaire regarding the cause(s) of their dyspnea, the Protocol Worksheet for
Pulmonary Hypertension (a standardized tool that is used in our Pulmonology Division), manovacuometer testing to determine the maximum inspiratory pressure (PImax) and maximum expiratory pressure (PEmax), and the 6MWT (completed on a continuous flat oval or rectangular track without obstacles and with $30 \mathrm{~m}$ markings).

Levels of anxiety and depression were calculated using the Hospital Anxiety and Depression Scale, which includes 14 questions regarding anxiety and depression. The scale scores range from 0 (anxiety/depression is improbable) to 21 (anxiety/ depression is probable). Scores of 0-7 were defined as anxiety/ depression being improbable, scores of 8-11 were defined as anxiety/depression being possible (questionable or doubtful), and scores of 12-21 were defined as anxiety/depression being probable ${ }^{8}$

Spirometry was performed using a portable computerized pulmonary testing system (Ferraris KOKO, Louisville, CO, USA), according to the criteria of the American Thoracic Society ${ }^{9}$

\section{Statistical analysis}

Categorical variables were compared using the chi-square test. The Kruskal-Wallis test was performed for asymmetrically distributed continuous variables when the sample size was relatively small (G1 and G3) and was followed by multiple comparisons using the Dunn method. Differences were considered statistically significant at a p-value of $<0.05$. All analyses were performed using Sigma Stat software (version 2.0).

\section{RESULTS}

This study evaluated 187 individuals, although only 107 patients were included in the analyses, based on the inclusion and exclusion criteria. The G1, G2, and G3 groups were compared according to their general characteristics (age, sex, race, weight, height, body mass index, and New York Health Association class) (Table 1). These comparisons revealed that the G1 group was younger than the other groups. Male patients were more common in all groups, compared to female patients. Among the 93 patients in the $\mathrm{G} 2$ group (Chagas disease without echocardiography findings suggestive of $\mathrm{PH}$ ), 53 patients had functional class I disease, which indicated that they did not have limitations. Among the 6 patients in the G3 group (Chagas disease with echocardiography findings suggestive of $\mathrm{PH}$ ), 5 patients had functional class II disease, which indicated that they had mild limitations (Table 1).

The G2 and G3 groups had similar values for real maximum inspiratory pressure (PImaxR), predicted maximum inspiratory pressure (PImaxP), real expiratory pressure (PEmaxR), and predicted maximum expiratory pressure (PEmaxP). The G1 group walked a median distance of $540 \mathrm{~m}$ (range: 510-619m) during the 6MWT, which was significantly farther than the distances for the G2 and G3 groups (Table 2). The predicted result for the 6MWT in the G1 group was significantly further than that in the G3 group ( $p=0.015)$.

There were no significant inter-group differences in the spirometry variables. However, the G3 group tended to 


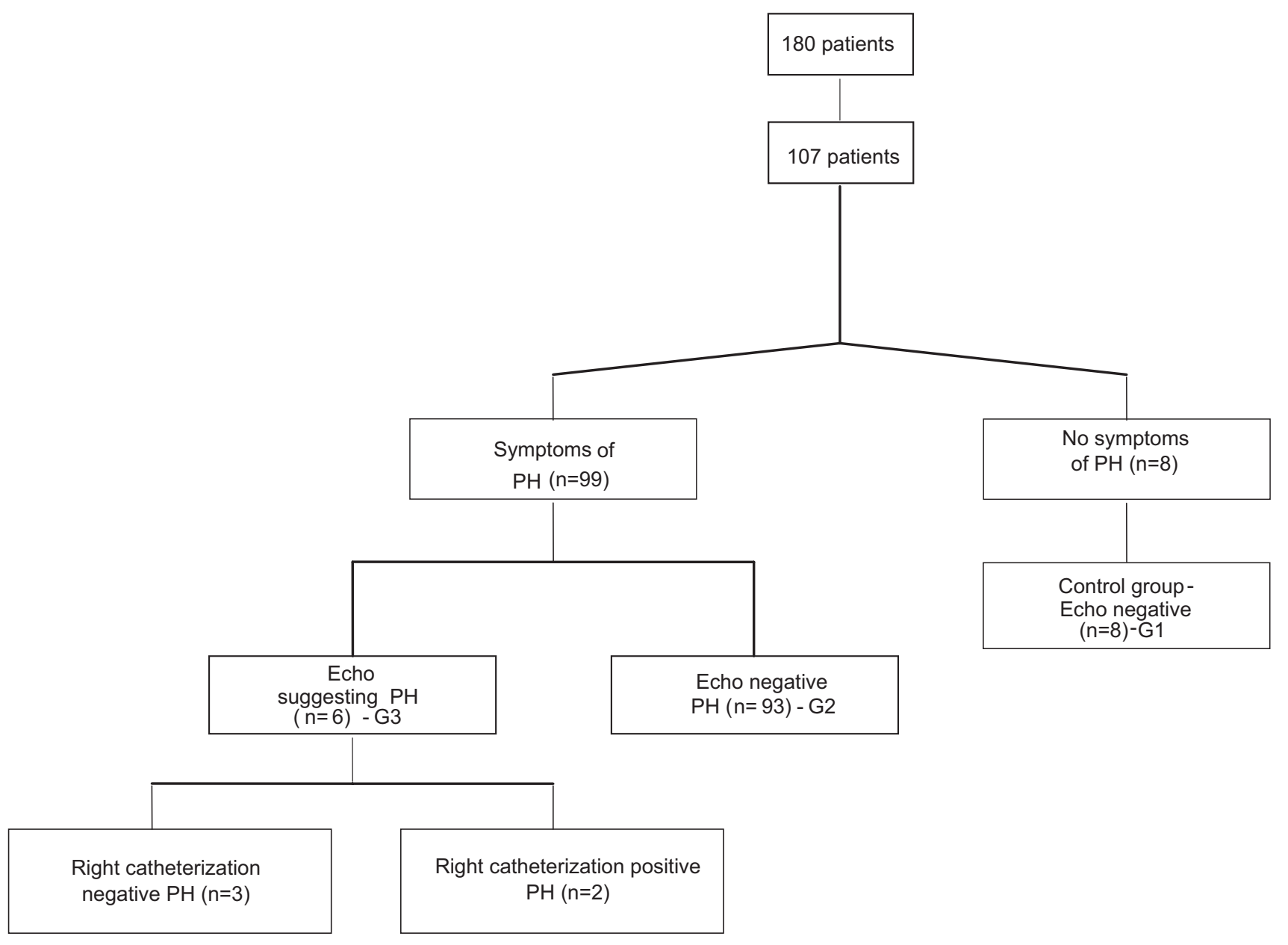

FIGURE 1 - The study flow chart. PH: pulmonary hypertension; Echo: echocardiography.

TABLE 1

The included patients' general characteristics.

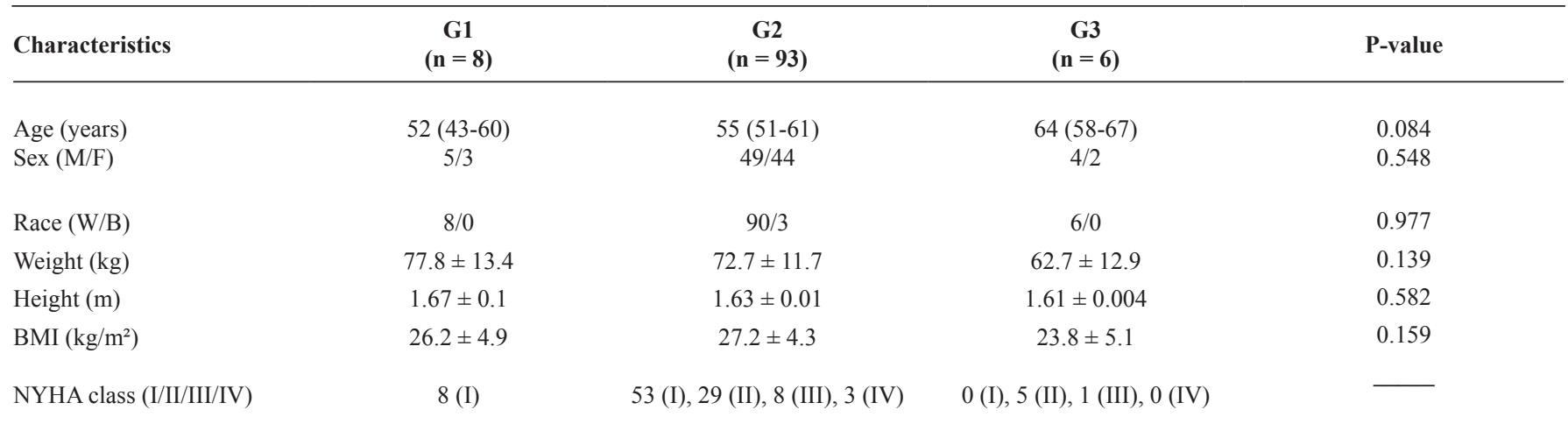

M: male; F: female; W: white; B: black; BMI: body mass index; NYHA: New York Health Association class. P-values were obtained using the chi-square test or the Kruskal-Wallis and Dunn's method. Values are expressed as mean \pm standard deviation or median (interquartile range). 
TABLE 2

Data from the 6-min walking test for the three groups.

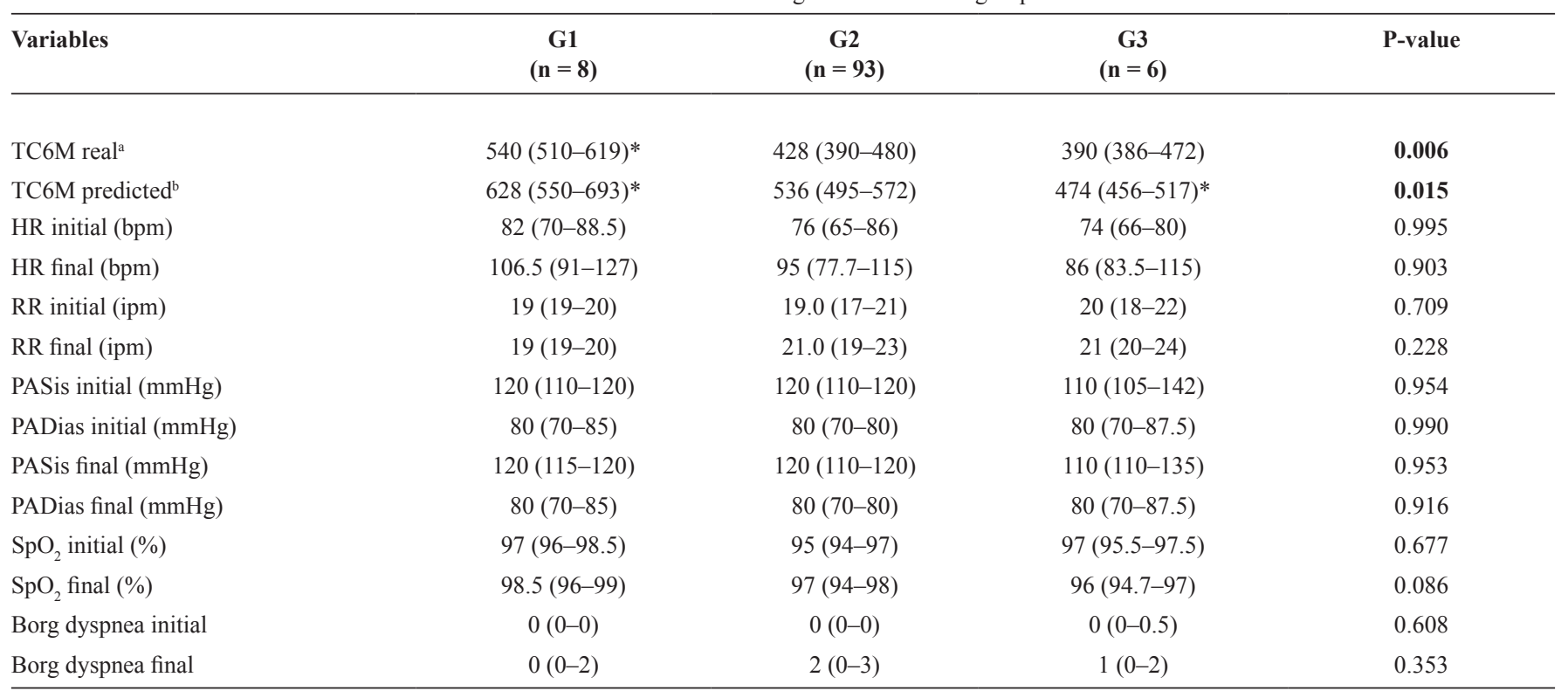

TC6M real $^{\mathbf{a}}$ : real 6-min walk test result ( $\mathrm{p}<0.05$, G1 vs. G2 and G3); Predicted ${ }^{\mathrm{b}}$ TC6M ( $<0.005$ G1 vs. G3); HR: heart rate; bpm: beats per minute;

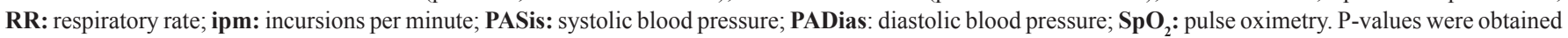
using the chi-square test or the Kruskal-Wallis and Dunn's method. Values are expressed as mean \pm standard deviation or median (interquartile range).

have lower values, compared to the other groups. The three groups had similar values for the Hospital Scale of Anxiety and Depression and SF-36 scores, and these results were not related to space limitations (Table 2).

\section{DISCUSSION}

Respiratory muscle weakness can be associated with increased respiratory system workload and reduced central or peripheral neural stimulation. In healthy individuals, the force exerted by the respiratory muscles must exceed the sum of the work that is performed by the lungs, thoracic case, and airways. Disequilibrium between the load and force (typically in the inspiratory muscles) results in a progressive muscle weakening that evolves towards alveolar hypoventilation and respiratory insufficiency. Thus, pathologies with cardiac impairment, especially cardiac and pulmonary insufficiency in obstructive or restrictive diseases, can affect the entire respiratory musculature. Therefore, it is important to evaluate these muscles to clarify the cause of symptoms (e.g., dyspnea) and clinical-functional disorders in patients with respiratory failure.

Previous studies have measured PImax and PEmax to confirm that weakness in the respiratory musculature is observed in various non- $\mathrm{PH}$ pathologies, such as congestive heart failure and chronic obstructive pulmonary disease (COPD). In the present study, we observed that the affected patients' respiratory musculature was weaker than the expected values for unaffected individuals. The G1 group had a relatively small difference between the predicted and measured values, although the G2 and G3 groups had more noticeable differences between the predicted and measured values. Nevertheless, as we did not detect any statistically significant differences in the present study, larger studies may be needed to provide additional evidence regarding this topic. In addition, a prospective study of 20 control patients and 37 patients with idiopathic PH (class II-IV, 26 women and 11 men) revealed that the patients with PH had lower values for PImax and PEmax, as well as weakness in the inspiratory and expiratory musculature ${ }^{10}$. These patients may hyperventilate during rest, exercise, and sleep, which can cause increased respiratory muscle activity, overload the inspiratory musculature, and lead to muscular weakness. Another experimental study evaluated changes in contractile performance, diaphragm morphology, and inspiratory muscle weakness in rats with $\mathrm{PH}$, and revealed that these rats had a significant reduction in the diaphragm's maximal contraction and generation forces. As this weakness appears to be specific to the diaphragm muscle, rather than generalized weakness, it is possible that chronic activity of the diaphragm can be triggered in cases of $\mathrm{PH}^{11,12}$.

The present study evaluated the patients' maximum respiratory pressures and 6MWT results based on their simplicity, practicality, efficacy, and validation for examining various pathologies. Compared to the G2 and G3 groups, the G1 group had a significantly greater distance during the $6 \mathrm{MWT}$ $(p=0.006)$, although the three groups had similar values for functional class, respiratory function, blood pressure, $\mathrm{SPO}_{2}$, and the Borg scale. According to the European Society of Cardiology and the European Respiratory Society, patients who can walk $>440 \mathrm{~m}$ have a low risk of poor outcomes, which corresponds to $5 \%$ between functional classes I and II. Distances of $165-440 \mathrm{~m}$ are associated with an intermediate risk, which corresponds 
to $5-10 \%$ in functional class III. Distances of $<165 \mathrm{~m}$ are associated with a high risk, which corresponds to $>10 \%$ in functional class $\mathrm{IV}^{13}$. Therefore, our patients in the G3 group had an intermediate risk (5-10\%), based on their 6MWT results (median: 390m, range: $386-472 \mathrm{~m}$ ), although 5 patients were functional class II and 1 patient was functional class III. It is possible that these patients, despite still being clinically stable relative to the control group (G1), require close monitoring with periodic clinical evaluations.

We used spirometry to evaluate pulmonary function, but did not detect any significant differences, although the G3 group tended to have lower values for the studied variables. Zanchet et al. ${ }^{14}$ assessed the effect of pulmonary rehabilitation on exercise capacity and respiratory muscle strength in 27 patients with COPD, and found that these patients walked $513 \mathrm{~m}$ during the $6 \mathrm{MWT}$. That result is noticeably greater than the results in our corresponding groups of patients (G2: 428m, $\mathrm{G} 3: 329 \mathrm{~m}$ ). Interestingly, the patients were clinically stable and did not have exacerbated disease (no changes in respiratory secretion, cough, fatigue, or dyspnea), which suggests that they had a better functional capacity that allowed them to cover greater distances. Thus, compared to patients with COPD, patients with PH may have lower functional capacity, which may contribute to their poor prognosis. In the study by Zanchet et al., the pre-rehabilitation PImax was $89 \mathrm{cmH}_{2} \mathrm{O}$ and the PEmax was $95 \mathrm{cmH}_{2} \mathrm{O}^{14}$, and those values were higher than the values that we observed (PImax: $84 \mathrm{cmH}_{2} \mathrm{O}$, PEmax: $70 \mathrm{cmH}_{2} \mathrm{O}$ ). These findings indicate that the patients had pulmonary functions that were below the predicted values for a healthy population.

We evaluated quality of life using the SF-36 questionnaire, which examines the patient's general health status. The three groups had similar values for all domains, and these findings are similar to those from a previous study of 34 patients who received 1 year of PAH therapy and completed the SF-36 questionnaire ${ }^{15}$. In that study, the patients walked $399 \mathrm{~m}$ during the 6MWT, which is similar to the mean value of $390 \mathrm{~m}$ in the present study. Although the patients' SF-36 scores remained stable, the greatest reductions were observed in functional capacity, physical abilities, and general health status ${ }^{5}$. We also observed that the lowest numerical values were observed for general health status. Thus, it appears that these patients are stable and their health-related quality of life is not necessarily associated with any limitation in exercise capacity that is caused by PAH, especially in the medium term. Nevertheless, it remains important to consider these patients' quality of life in future studies of PAH, given that their clinical condition can affect both the patient and their families.

In conclusion, we observed that patients with indeterminate chronic Chagas disease and symptoms of $\mathrm{PH}$ did not appear to experience impairments in their functioning, based on our results regarding their respiratory musculature, spirometry, and Hospital Anxiety and Depression Scale scores. Nevertheless, these patients appear to have low exercise tolerance, based on the 6MWT results.

\section{Acknowledgments}

We thank the Botucatu Medical School for providing technical support for the development and implementation of this study.

\section{Financial support}

Coordenação de Aperfeiçoamento de Pessoal de Nível Superior (CAPES).

\section{Conflict of interest}

The authors declare that there is no conflict of interest.

\section{REFERENCES}

1. Hoeper MM, Bogaard HJ, Condliffe R, Frantz R, Khanna D, Kurzyna M, et al. Definitions and diagnosis of pulmonary hypertension. J Am Coll Cardiol. 2013;62(suppl 25):D42-50.

2. Kabitz HJ, Bremer HC, Schwoerer A, Sonntag F, Walterspacher $\mathrm{S}$, Walker DJ, et al. The combination of exercise and respiratory training improves respiratory muscle function in pulmonary hypertension. Lung. 2014;192(2):321-8.

3. Diretrizes Brasileiras para Manejo da Hipertensão Pulmonar 2005. Classificação e avaliação diagnóstica da hipertensão pulmonar. J Bras Pneumol. 2005;31(suppl 2):S1-S8.

4. Rassi Jr A, Rassi A, Rassi SG. Predictors of mortality in chronic Chagas disease: a systematic review of observational studies. Circulation. 2007;115(9):1101-8.

5. Dias JCP, Ramos Jr ANJ, Gontijo ED, Luquetti A, Shikanai-Yasuda MA, Coura JR, et al. II Consenso Brasileiro em Doença de Chagas, 2015. Epidemiol Serv Saúde. 2016;25(esp):7-86.

6. Bilate AM, Cunha-Neto E. Chagas disease cardiomyopathy: current concepts of an old disease. Rev Inst Med Trop São Paulo. 2008;50(2):67-74.

7. Prata A. Clinical and epidemiological aspects of Chagas disease. Lancet Infect Dis. 2001;1(2):92-100.

8. Zigmond AS, Snaith RP. The hospital anxiety and depression scale. Acta Psychiatr Scand. 1983;67(6):361-70.

9. Miller MR, Hankinson J, Brusasco V, Bungos F, Casabrini R, Coates A, et al. ATS/ERS Task Force. Standardisation of spirometry. Eur Respir J. 2005; 26:319-38.

10. Meyer FJ, Lossnitzer D, Kristen AV, Schoene AM, Kübler W, Katus HA, et al. Respiratory muscle dysfunction in idiopathic pulmonary arterial hypertension. Eur Respir J. 2005;25(1):125-30.

11. de Man SF, van Hees HW, Handoko LM, Niessen HW, Schalij I, Humbert M, et al. Diaphragm muscle fiber weakness in pulmonary hypertension. Am J Respir Crit Care Med. 2011;183(10):1411-8.

12. Kabitz JH, Schwoerer A, Bremer HC, Sonntag F, Walterspacher $\mathrm{S}$, Walker D, et al. Impairment of respiratory muscle function in pulmonary hypertension. Clin Sci. 2008;114(2):165-71.

13. Galiè N, Humbert M, Vachiery JL, Gibbs S, Lang I, Torbicki A, et al. $2015 \mathrm{ESC} / \mathrm{ERS}$ Guidelines for the diagnosis and treatment of pulmonary hypertension: the joint task force for the diagnosis and treatment of pulmonary hypertension of the european society of cardiology (ESC) and the European Respiratory Society (ERS): endorsed by: Association for European Paediatric and Congenital Cardiology (AEPC), International Society for Heart and Lung Transplantation (ISHLT). Eur Respir J. 2015;46(4):903-75.

14. Zanchet CR, Viegas AAC, Lima T. Efficacy of pulmonary rehabilitation: exercise capacity, respiratory muscle strength and quality of life in patients with chronic obstructive pulmonary disease. J Bras Pneumol. 2005;31(2):118-24.

15. Cícero C, Franchi MS, Barreto CA, Lopes BAA. Lack of tight association between quality of life and exercise capacity in pulmonary arterial hypertension. Arq Bras Cardiol. 2012;99(4):876-85. 